\title{
Exact discrete sampling of finite variation tempered stable Ornstein-Uhlenbeck processes
}

\author{
Reiichiro Kawai and Hiroki Masuda
}

\begin{abstract}
Exact yet simple simulation algorithms are developed for a wide class of Ornstein-Uhlenbeck processes with tempered stable stationary distribution of finite variation with the help of their exact transition probability between consecutive time points. Random elements involved can be divided into independent tempered stable and compound Poisson distributions, each of which can be simulated in the exact sense through acceptance-rejection sampling, respectively, with stable and gamma proposal distributions. We discuss various alternative simulation methods within our algorithms on the basis of acceptance rate in acceptance-rejection sampling for both high- and low-frequency sampling. Numerical results illustrate their advantage relative to the existing approximative simulation method based on infinite shot noise series representation.
\end{abstract}

Keywords. Acceptance-rejection sampling, high-frequency sampling, Lévy process, Ornstein-Uhlenbeck process, subordinator, transition probability, tempered stable process.

2010 Mathematics Subject Classification. 68U20, 62E15, 65C10, 60E07.

\section{Introduction}

The class of non-Gaussian Ornstein-Uhlenbeck processes has long been of both theoretical and practical interest. From a theoretical point of view, on one hand, this class is closely related to the self-decomposable infinitely divisible distribution. Several interesting properties are known, such as the explicit relation between the Lévy measures of the stationary distribution and the underlying Lévy process and the representation of entire trajectory using the series representation of underlying Lévy process, to mention just a few. (For details, see Section 17 of Sato [21], Masuda [18] and references therein.) On the other hand, in practice, non-Gaussian Ornstein-Uhlenbeck processes have been used in mathematical physics under the name of exponentially correlated colored noise, and more recently in financial economics and mathematical finance (for example, Barndorff-Nielsen and Shephard [3,4] and Benth et al. [5]). Due to the growing practical interest, many authors have proposed statistical inference methods for non-Gaussian Ornstein-Uhlenbeck processes. (See, for example, Brockwell et al. [7] and Jongbloed et al. [13].) 
The main purpose of this paper is to develop and investigate exact simulation algorithms for a wide class of Ornstein-Uhlenbeck processes of finite variation with tempered stable stationary laws. In particular, the flexibility and mathematical tractability of the tempered stable distribution makes this class more attractive than the other classes of Ornstein-Uhlenbeck processes. It is well known that the exact simulation of its entire trajectory over a finite horizon is only possible, in principle, with the infinite series representation of tempered stable Lévy processes. (See, for example, Barndorff-Nielsen and Shephard [3,4] and Rosiński [20].) This method often requires extremely expensive computing effort, especially when the convergence of the infinite series is very slow. In addition, simulation via the series representation is no longer an exact method as soon as the infinite sum is truncated. The exact simulation algorithm we develop in this paper is designed to generate arbitrary discrete time skeleton of the trajectory. Thanks to the homogeneous Markovian autoregressive structure of Ornstein-Uhlenbeck processes, its transition probability between consecutive times can be derived in closed form, in a similar manner to Zhang and Zhang [23,24]. Random elements involved can be divided into independent tempered stable and compound Poisson components, each of which can be simulated exactly with acceptance-rejection sampling, respectively, with non-tempered stable and gamma proposal distributions. It turns out that our approach is easily applicable to the settings of bilateral tempered stable Ornstein-Uhlenbeck processes of finite variation and of normal tempered stable processes as well.

The rest of this paper is organized as follows. Section 2 summarizes background material on stable and tempered stable subordinators and on tempered stable Ornstein-Uhlenbeck processes. In Section 3, we derive the exact transition probability consisting of independent tempered stable and compound Poisson components. In Section 4, acceptance-rejection sampling methods are discussed for tempered stable and compound Poisson distributions for different sampling frequencies. In particular, we discuss various alternative simulation methods within our algorithms on the basis of acceptance rate in acceptance-rejection sampling. We provide in Section 5 numerical results to illustrate the effectiveness of our exact simulation algorithms relative to the existing approximative method based on infinite series representation. Finally, Section 6 concludes.

\section{Preliminaries}

Let us begin this preliminary section with the notation which will be used throughout the paper. We denote by $\mathbb{R}$ the one dimensional Euclidean space with the norm $|\cdot|, \mathbb{R}_{+}:=(0,+\infty)$ and $\mathbb{R}_{-}:=(-\infty, 0)$. Let $\mathbb{N}$ be the collection of positive 
integers with $\mathbb{N}_{0}:=\mathbb{N} \cup\{0\}$. We denote by $\mathscr{L}(X)$ and $\stackrel{\mathscr{L}}{=}$, respectively, the law of random variable $X$ and identity in law. We denote by $\Gamma(a, b)$ the gamma distribution with density $b^{a} / \Gamma(a) x^{a-1} e^{-b x}$. We fix $(\Omega, \mathcal{F}, \mathbb{P})$ as our underlying probability space. We say that the stochastic process $\left\{Y_{t}: t \geq 0\right\}$ in $\mathbb{R}$ is a subordinator (without drift) if it is a non-decreasing Lévy process with characteristic function

$$
\mathbb{E}\left[e^{i y Y_{t}}\right]=\exp \left[t \int_{\mathbb{R}_{+}}\left(e^{i y z}-1\right) v(d z)\right],
$$

where $v$ is a Lévy measure defined on $\mathbb{R}_{+}$satisfying $\int_{0}^{1} z v(d z)<+\infty$. Finally, let us note that $\Gamma(-s)<0$ for $s \in(0,1)$.

\subsection{Stable subordinator}

Let $\left\{L_{t}^{(\mathrm{s})}: t \geq 0\right\}$ be a stable subordinator with characteristic function

$$
\begin{aligned}
\mathbb{E}\left[e^{i y L_{t}^{(\mathrm{s})}}\right] & =\exp \left[t \int_{\mathbb{R}_{+}}\left(e^{i y z}-1\right) \frac{a}{z^{\alpha+1}} d z\right] \\
& =\exp \left[\operatorname{ta} \Gamma(-\alpha) \cos \left(\frac{\pi \alpha}{2}\right)|y|^{\alpha}\left(1-i \tan \frac{\pi \alpha}{2} \operatorname{sgn}(y)\right)\right],
\end{aligned}
$$

with $\alpha \in(0,1)$ and $a>0$. Note that $a$ only acts as a scale parameter. For each $t>0$, the marginal $L_{t}^{(\mathrm{s})}$ has a stable distribution on $\mathbb{R}_{+}$and $\mathbb{E}\left[\left(L_{t}^{(\mathrm{s})}\right)^{\theta}\right]$ is finite if $\theta \in(0, \alpha)$, while is infinite if $\theta \geq \alpha$. Throughout this paper, we denote by $S(\alpha, a)$ the distribution of $L_{1}^{(\mathrm{s})}$ when (2.2) is satisfied. Clearly, it holds that for each $t>0$, $\mathscr{L}\left(L_{t}^{(\mathrm{s})}\right)=S(\alpha, t a)$. The distribution $S(\alpha, a)$ can be simulated in the exact sense through the well known representation, due to Kanter [14] and Chambers et al. [8],

$$
S(\alpha, a) \stackrel{\mathscr{L}}{=}\left(\frac{a \Gamma(1-\alpha)}{\alpha \cos (V)}\right)^{\frac{1}{\alpha}} \sin (\alpha(V+\pi / 2))\left(\frac{\cos (V-\alpha(V+\pi / 2))}{E}\right)^{\frac{1-\alpha}{\alpha}},
$$

where $V$ is a uniform random variable on $(-\pi / 2, \pi / 2)$ and $E$ is a standard exponential random variable independent of $V$. The distribution $S(\alpha, a)$ admits $C^{\infty}$ density on $\mathbb{R}_{+}$given in form of convergent series

$$
\begin{aligned}
f_{S(\alpha, a)}(x):= & \frac{1}{\pi(-a \Gamma(-\alpha))^{1 / \alpha}} \\
& \times \sum_{k=1}^{+\infty}(-1)^{k-1} \sin (k \pi \alpha) \frac{\Gamma(k \alpha+1)}{k !}\left(\frac{x}{(-a \Gamma(-\alpha))^{1 / \alpha}}\right)^{-k \alpha-1} .
\end{aligned}
$$

See Zolotarev [25] for more details on the stable distribution. 


\subsection{Tempered stable subordinator}

Consider the exponentially tempered stable Lévy density

$$
v(z)=a \frac{e^{-b z}}{z^{\alpha+1}}, \quad z \in \mathbb{R}_{+},
$$

where $a>0, b>0$ and $\alpha \in(0,1)$. The associated subordinator

$$
\left\{L_{t}^{(\mathrm{ts})}: t \geq 0\right\}
$$

(without drift) is often called the tempered stable subordinator, with characteristic function

$$
\begin{aligned}
\mathbb{E}\left[e^{i y L_{t}^{(\mathrm{ts})}}\right] & =\exp \left[t \int_{\mathbb{R}_{+}}\left(e^{i y z}-1\right) v(z) d z\right] \\
& =\exp \left[\operatorname{ta} \Gamma(-\alpha)\left((b-i y)^{\alpha}-b^{\alpha}\right)\right] .
\end{aligned}
$$

Throughout this paper, we denote by $\operatorname{TS}(\alpha, a, b)$ the distribution of $L_{1}^{(\mathrm{ts})}$ defined on $\mathbb{R}_{+}$, which we call tempered stable distribution, when (2.6) is satisfied. Clearly, it holds that for each $t>0$,

$$
\mathscr{L}\left(L_{t}^{(\mathrm{ts})}\right)=\operatorname{TS}(\alpha, t a, b) .
$$

The tempered stable distribution admits $C^{\infty}$-density on $\mathbb{R}_{+}$as well, with a simple yet very insightful relation to the density of its non-tempered stable distribution

$$
f_{\mathrm{TS}(\alpha, a, b)}(x):=e^{-b x-a \Gamma(-\alpha) b^{\alpha}} f_{S(\alpha, a)}(x) .
$$

This property acts as a key building block later. The class of tempered stable distributions is first proposed by Tweedie [22]. Barndorff-Nielsen and Shephard [4] studies the tempered stable subordinator and the so-called normal tempered stable law, that is, a normal variance-mean mixture of the positive tempered stable distribution, with a view towards financial economics. Various featuring properties of tempered stable processes are revealed by Rosiński [20], such as a stable-like behavior over short intervals, the absolute continuity with respect to its short-range limiting stable subordinator (Proposition 4.1), aggregational Gaussianity and an infinite series representation in closed form

$$
\begin{aligned}
\left\{L_{t}^{(\mathrm{ts})}:\right. & t \in[0, T]\} \\
& \stackrel{\mathscr{L}}{=}\left\{\sum_{k=1}^{+\infty}\left[\left(\frac{\alpha \Gamma_{k}}{a T}\right)^{-1 / \alpha} \wedge \frac{V_{k} U_{k}^{1 / \alpha}}{b}\right] \mathbf{1}\left(T_{k} \in[0, t]\right): t \in[0, T]\right\},
\end{aligned}
$$


which was first introduced by Rosiński in the discussion part of Barndorff-Nielsen and Shephard [3]. Here, $\left\{\Gamma_{k}\right\}_{k \in \mathbb{N}}$ denotes a sequence of standard Poisson arrivals, $\left\{T_{k}\right\}_{k \in \mathbb{N}}$ is a sequence of iid uniform random variables on $[0, T],\left\{V_{k}\right\}_{k \in \mathbb{N}}$ is a sequence of iid standard exponential random variables and $\left\{U_{k}\right\}_{k \in \mathbb{N}}$ is a sequence of iid uniform random variables on $[0,1]$. All those random sequences are mutually independent. Note that the kernel of series representation is not unique. (See Imai and Kawai $[11,12]$ for different representations and numerical issues.)

\subsection{Ornstein-Uhlenbeck Processes with tempered stable stationary distribution}

Consider the stochastic process $\left\{Y_{t}: t \geq 0\right\}$ defined in form of stochastic differential equation

$$
d Y_{t}=-\lambda Y_{t} d t+d Z_{\lambda t}
$$

where $\lambda>0$ and $\left\{Z_{t}: t \geq 0\right\}$ is a subordinator, or in canonical form

$$
Y_{t}=e^{-\lambda t} Y_{0}+e^{-\lambda t} \int_{0}^{\lambda t} e^{s} d Z_{s}
$$

The process of this type is called a Lévy-driven Ornstein-Uhlenbeck process and is used, for example, to model the squared volatility in a stochastic volatility model (Barndorff-Nielsen and Shephard [3]).

The Lévy density (2.5) forms a self-decomposable Lévy measure. By the arguments in Section 17 of Sato [21], there exists an Ornstein-Uhlenbeck process $\left\{Y_{t}: t \geq 0\right\}$ whose marginal has the infinitely divisible distribution with the tempered stable Lévy density (2.5), if the initial state $Y_{0}$ is chosen to have the same distribution to the stationary infinitely divisible distribution. In particular, the Ornstein-Uhlenbeck process with inverse Gaussian stationary marginal $(\alpha=1 / 2)$ is often abbreviated to IG-OU and is applied in Benth [5] to stochastic volatility modeling of [3] for volatility and variance swap valuations.

Let $w(z)$ be the Lévy density of the marginal $Z_{1}$ and let $u(z)$ be the Lévy density of the stationary marginal $Y_{1}$. If $u(z)$ is differentiable, then the Lévy densities $w(z)$ and $u(z)$ are related as

$$
w(z)=-u(z)-z \frac{\partial}{\partial z} u(z)=a\left(\frac{\alpha}{z}+b\right) \frac{e^{-b z}}{z^{\alpha}} .
$$

This implies that the underlying subordinator $\left\{Z_{t}: t \geq 0\right\}$ is the superposition of a tempered stable subordinator and a compound Poisson process. With the help of the infinite shot noise series representation (2.8) of tempered stable subordinators, 
we can formulate sample paths as

$$
\begin{aligned}
& \left\{Y_{t}: t \in[0, T]\right\} \\
& \stackrel{\mathscr{L}}{=}\left\{e^{-\lambda t} Y_{0}+\sum_{k=1}^{+\infty} e^{\lambda\left(T_{k}-t\right)}\left[\left(\frac{\Gamma_{k}}{a T}\right)^{-1 / \alpha} \wedge \frac{V_{k} U_{k}^{1 / \alpha}}{b}\right] \mathbf{1}\left(T_{k} \in[0, t]\right)\right. \\
& \left.\quad+\sum_{k=1}^{+\infty} e^{\widetilde{\Gamma}_{k}-\lambda t} G_{k} \mathbf{1}\left(\widetilde{\Gamma}_{k} \in[0, \lambda t]\right): t \in[0, T]\right\},
\end{aligned}
$$

where $\left\{\widetilde{\Gamma}_{k}\right\}_{k \in \mathbb{N}}$ is a sequence of Poisson arrivals with intensity $a \Gamma(1-\alpha) b^{\alpha}$, independent of $\left\{\Gamma_{k}\right\}_{k \in \mathbb{N}}$, and $\left\{G_{k}\right\}_{k \in \mathbb{N}}$ is a sequence of iid random variables with gamma distribution $\Gamma(1-\alpha, b)$.

In fact, we can readily extend to the bilateral finite variation setting by superpositioning two independent subordinators in the opposite directions, by setting $Z_{t}:=Z_{t}^{+}-Z_{t}^{-}$in the definition (2.9) or (2.10), where $\left\{Z_{t}^{ \pm}: t \geq 0\right\}$ are independent subordinators with suitable laws. This setting will be considered in Corollary 3.2 .

\section{Exact transition probability}

The main purpose of this paper is to develop an exact simulation algorithm for arbitrary discrete time skeleton

$$
Y_{0}, Y_{\Delta}, Y_{2 \Delta}, \ldots
$$

of the tempered stable Ornstein-Uhlenbeck process (2.9), with a positive stepsize $\Delta$. (In principle, discrete observations do not need to be equidistant. Stepsizes can be set different positive values for different steps.) To this end, we first provide the exact transition probability of the random sequence $\left\{Y_{k \Delta}\right\}_{k \in \mathbb{N}_{0}}$, due to Zhang and Zhang [24]. For completeness, we also outline its proof.

Theorem 3.1. For each $n \in \mathbb{N}_{0}$, it holds that given $Y_{n \Delta}$,

$$
Y_{(n+1) \Delta} \stackrel{\mathscr{L}}{=} e^{-\lambda \Delta} Y_{n \Delta}+\eta_{0}(\Delta)+\sum_{k=1}^{N(\Delta)} \eta_{k}(\Delta),
$$

where $N(\Delta)$ and $\eta_{0}(\Delta), \eta_{1}(\Delta), \ldots$ are independent random variables specified as follows:

- $\eta_{0}(\Delta) \sim \operatorname{TS}\left(\alpha, a\left(1-e^{-\alpha \lambda \Delta}\right), b\right)$.

- $N(\Delta)$ denotes the Poisson random variable with intensity

$$
-a\left(1-e^{-\alpha \lambda \Delta}\right) \Gamma(-\alpha) b^{\alpha} .
$$


- $\left\{\eta_{k}(\Delta)\right\}_{k \in \mathbb{N}}$ is a sequence of iid random variables with common probability density

$$
v_{\Delta}(x):=\frac{1}{\left(1-e^{\alpha \lambda \Delta}\right) \Gamma(-\alpha) b^{\alpha}} x^{-1-\alpha}\left(e^{-b x}-e^{-b e^{\lambda \Delta} x}\right), \quad x \in \mathbb{R}_{+} .
$$

Proof. By the homogeneous Markovian autoregressive structure of (2.10), it holds that for each $n \in \mathbb{N}_{0}$,

$$
\begin{aligned}
Y_{(n+1) \Delta} & =e^{-\lambda \Delta} Y_{n \Delta}+\int_{n \Delta}^{(n+1) \Delta} e^{-\lambda((n+1) \Delta-s)} d Z_{\lambda s} \\
& =: e^{-\lambda \Delta} Y_{n \Delta}+\epsilon_{\Delta, n+1} \\
& \stackrel{\mathscr{L}}{=} e^{-\lambda \Delta} Y_{n \Delta}+\int_{0}^{\lambda \Delta} e^{-\lambda \Delta+s} d Z_{s},
\end{aligned}
$$

where the identity in law holds by independence and stationarity of increments of the underlying subordinator $\left\{Z_{t}: t \geq 0\right\}$. This implies that $\left\{\epsilon_{\Delta, k}\right\}_{k \in \mathbb{N}}$ is simply a sequence of iid random variables with common distribution

$$
F_{\Delta}:=\mathscr{L}\left(\int_{0}^{\lambda \Delta} e^{-\lambda \Delta+s} d Z_{s}\right) .
$$

It thus suffices to investigate the conditional distribution $\mathscr{L}\left(Y_{\Delta} \mid Y_{0}\right)$ of the first increment. Note that by definition, this distribution is infinitely divisible.

Let $w(z)$ be the Lévy density of $Z_{1}$ given by (2.11). By the Lévy-integral transform of the characteristic function, we get

$$
\begin{aligned}
\ln \mathbb{E}\left[e^{i y \epsilon_{\Delta, 1}}\right] & =\int_{0}^{\lambda \Delta} \ln \mathbb{E}\left[e^{i y e^{-\lambda \Delta+s} Z_{1}}\right] d s \\
& =\int_{\mathbb{R}_{+}}\left(e^{i y z}-1\right)\left(\int_{0}^{\lambda \Delta} e^{s} w\left(e^{s} z\right) d s\right) d z \\
& =: \int_{\mathbb{R}_{+}}\left(e^{i y z}-1\right) w_{\Delta}(z) d z .
\end{aligned}
$$

Note that $w_{\Delta}(z)$ indicates the Lévy density of the distribution $F_{\Delta}$. Observe that for each $z \in \mathbb{R}_{+}$,

$$
\begin{aligned}
w_{\Delta}(z) & =a z^{-1-\alpha} \int_{0}^{\lambda \Delta}\left(\alpha+b e^{s} z\right) e^{-\alpha s} e^{-b e^{s} z} d s \\
& =a z^{-1-\alpha}\left(e^{-b z}-e^{-\alpha \lambda \Delta} e^{-b e^{\lambda \Delta} z}\right) \\
& =a\left(1-e^{-\alpha \lambda \Delta}\right) z^{-1-\alpha} e^{-b z}+a e^{-\alpha \lambda \Delta} z^{-1-\alpha}\left(e^{-b z}-e^{-b e^{\lambda \Delta} z}\right) \\
& =: w_{\Delta, 1}(z)+w_{\Delta, 2}(z),
\end{aligned}
$$


where the second equality holds by

$$
(\partial / \partial s)\left(-e^{-\alpha s} e^{-b e^{s} z}\right)=\left(\alpha+b e^{s} z\right) e^{-\alpha s} e^{-b e^{s} z} .
$$

Clearly, the function $w_{\Delta, 1}(z)$ is the Lévy density of $\operatorname{TS}\left(\alpha, a\left(1-e^{-\alpha \lambda \Delta}\right), b\right)$. Moreover, since

$$
\int_{\mathbb{R}_{+}} w_{\Delta, 2}(z) d z=a\left(e^{-\alpha \lambda \Delta}-1\right) \Gamma(-\alpha) b^{\alpha}<+\infty,
$$

the function $w_{\Delta, 2}(z)$ acts as the Lévy density of the compound Poisson component. This completes the proof.

Let us below describe a direct extension to the bilateral finite variation setting. We omit the proof to avoid overloading the paper with lengthy details of routine nature.

Corollary 3.2. For each $n \in \mathbb{N}_{0}$, it holds that given $Y_{n \Delta}$,

$$
Y_{(n+1) \Delta} \stackrel{\mathscr{L}}{=} e^{-\lambda \Delta} Y_{n \Delta}+\eta_{0}^{+}(\Delta)+\sum_{k=1}^{N^{+}(\Delta)} \eta_{k}^{+}(\Delta)-\eta_{0}^{-}(\Delta)+\sum_{k=1}^{N^{-}(\Delta)} \eta_{k}^{-}(\Delta),
$$

where $N^{+}(\Delta), N^{-}(\Delta), \eta_{0}^{+}(\Delta), \eta_{1}^{+}(\Delta), \ldots, \eta_{0}^{-}(\Delta), \eta_{1}^{-}(\Delta), \ldots$ are mutually independent random variables specified as follows:

- we have

$$
\eta_{0}^{+}(\Delta) \sim \operatorname{TS}\left(\alpha_{+}, a_{+}\left(1-e^{-\alpha_{+} \lambda \Delta}\right), b_{+}\right)
$$

and

$$
\eta_{0}^{-}(\Delta) \sim \operatorname{TS}\left(\alpha_{-}, a_{-}\left(1-e^{-\alpha_{-} \lambda \Delta}\right), b_{-}\right) .
$$

- $N^{+}(\Delta)$ and $N^{-}(\Delta)$ are Poisson random variables with intensities

$$
a_{+}\left(e^{-\alpha_{+} \lambda \Delta}-1\right) \Gamma\left(-\alpha_{+}\right) b_{+}^{\alpha_{+}} \text {and } a_{-}\left(e^{-\alpha_{-} \lambda \Delta}-1\right) \Gamma\left(-\alpha_{-}\right) b_{-}^{\alpha_{-}},
$$

respectively.

- $\left\{\eta_{k}^{+}(\Delta)\right\}_{k \in \mathbb{N}}$ and $\left\{\eta_{k}^{-}(\Delta)\right\}_{k \in \mathbb{N}}$ are sequences of iid random variables with common probability densities

$$
v_{\Delta}^{+}(x):=\frac{1}{\left(1-e^{\alpha+\lambda \Delta}\right) \Gamma\left(-\alpha_{+}\right) b_{+}^{\alpha_{+}}} x^{-1-\alpha_{+}}\left(e^{-b_{+} x}-e^{-b_{+} e^{\lambda \Delta_{x}}}\right)
$$

for $x \in \mathbb{R}_{+}$, and

$$
v_{\Delta}^{-}(x):=\frac{1}{\left(1-e^{\alpha_{-} \lambda \Delta}\right) \Gamma\left(-\alpha_{-}\right) b_{-}^{\alpha_{-}}}|x|^{-1-\alpha_{-}}\left(e^{-b_{-}|x|}-e^{-b_{-} e^{\lambda \Delta}|x|}\right)
$$

for $x \in \mathbb{R}_{-}$, respectively. 


\section{Exact simulation using acceptance-rejection sampling}

Due to the exact transitions of Theorem 3.1 and Corollary 3.2, the exact simulation of random elements involved enables one to simulate exactly the discrete time skeleton $\left\{Y_{k \Delta}\right\}_{k \in \mathbb{N}}$ in a recursive manner. Random elements to be generated are the tempered stable random variable $\eta_{0}(\Delta)$ and the random variables $N(\Delta)$ and $\left\{\eta_{k}(\Delta)\right\}_{k \in \mathbb{N}}$ in the compound Poisson component.

Let us begin with the exact simulation of $\eta_{0}(\Delta) \sim \operatorname{TS}\left(\alpha, a\left(1-e^{-\alpha \lambda \Delta}\right), b\right)$. An efficient exact simulation method for the case $\alpha=0.5$, that is the inverse Gaussian, is well known due to Michael et al. [19]. For the general case $\alpha \in(0,1)$, a straightforward approach would be to apply acceptance-rejection sampling based on the representation (2.3) of the stable distribution and the ratio (2.7) of the two densities, that is, for each $x \in \mathbb{R}_{+}$,

$$
\frac{f_{\mathrm{TS}(\alpha, a, b)}(x)}{f_{S(\alpha, a)}(x)}=e^{-b x-a \Gamma(-\alpha) b^{\alpha}} \leq e^{-a \Gamma(-\alpha) b^{\alpha}} .
$$

(See, for example, Brix [6].) The acceptance-rejection sampling algorithm for generation of the random variable $\eta_{0}(\Delta)$ is then as simple as

\section{Algorithm 1.}

Step 1 Generate $U$ as uniform $(0,1)$ and $V$ as $S\left(\alpha, a\left(1-e^{-\alpha \lambda \Delta}\right)\right)$ through (2.3).

Step 2 If $U \leq e^{-b V}$, let $\eta_{0}(\Delta) \leftarrow V$. Otherwise, return to Step 1.

Note that this algorithm clearly works more efficiently when the acceptance rate $e^{a\left(1-e^{-\alpha \lambda \Delta}\right) \Gamma(-\alpha) b^{\alpha}}$ at Step 2 is closer to 1 . This happens when $b \downarrow 0$ and/or $\Delta \downarrow 0$. The case $b \downarrow 0$ is obvious since then the tempered stable distribution approaches to its stable proposal distribution. In practice, we only have control on the time interval $\Delta$. To account for the case $\Delta \downarrow 0$, we employ the short-range behavior of tempered stable subordinators, which is rigorously proved first by Rosiński [20].

Proposition 4.1. Let $\left\{L_{t}^{(\mathrm{s})}: t \geq 0\right\}$ and $\left\{L_{t}^{(\mathrm{ts})}: t \geq 0\right\}$ be Lévy processes respectively with $S(\alpha, a)$ and $\operatorname{TS}(\alpha, a, b)$. It holds that as $h \downarrow 0, h>0$,

$$
\left\{h^{-1 / \alpha} L_{h t}^{(\mathrm{ts})}: t \geq 0\right\} \rightarrow\left\{L_{t}^{(\mathrm{s})}: t \geq 0\right\},
$$

where the convergence of random processes holds in the weak sense in the space $\mathbb{D}\left([0,+\infty) ; \mathbb{R}_{+}\right)$of càdlàg functions equipped with the Skorohod topology.

This convergence result implies that a tempered stable marginal over a very short time is very close to a stable distribution. The acceptance rate thus tends 
to 1 as well. To illustrate this phenomenon, we compare in Table 1 percentiles of a tempered stable marginal and its stable proposal marginal at time $t=0.1$, 0.01 and 0.001 . The percentiles are estimated by Monte Carlo methods based on 3000000 iid replications. Acceptance rates of acceptance-rejection sampling are respectively $0.7192,0.9676$ and 0.9967 . Clearly, the tempered stable distribution tends to the stable proposal distribution as $t$ is smaller. It is worth pointing out an obvious merit of Algorithm 1 in the implementation of the Euler-Maruyama scheme for more general stochastic differential equations driven by a tempered stable subordinator, in which stepsize $\Delta$ is often desired to be taken arbitrarily small.

\begin{tabular}{|c||c|c|c|c|c|c|c|c|c|}
\hline & $20 \%$ & $40 \%$ & $60 \%$ & $80 \%$ & $90 \%$ & $95 \%$ & $97 \%$ & $98 \%$ & $99 \%$ \\
\hline \hline$L_{1}^{(\mathrm{s})}$ & 5.25 & 6.77 & 9.29 & 16.43 & 31.51 & 65.17 & 115.65 & 186.04 & 425.94 \\
\hline \hline$h_{1}^{-1 / \alpha} L_{h_{1}}^{(\mathrm{ts})}$ & 5.25 & 6.76 & 9.27 & 16.31 & 30.92 & 62.44 & 107.76 & 167.31 & 350.53 \\
\hline$h_{2}^{-1 / \alpha} L_{h_{2}}^{(\mathrm{ts})}$ & 5.22 & 6.68 & 9.05 & 15.36 & 27.07 & 48.73 & 74.51 & 103.28 & 172.17 \\
\hline$h_{3}^{-1 / \alpha} L_{h_{3}}^{\text {(ts) }}$ & 5.00 & 6.19 & 7.89 & 11.47 & 16.33 & 22.76 & 28.52 & 33.73 & 44.02 \\
\hline
\end{tabular}

Table 1. Percentile comparison of scaled marginals $h^{-1 / \alpha} L_{h}^{\text {(ts) }}$ of $\operatorname{TS}(0.8,1.0,0.5)$ and the 0.8 -stable proposal distribution $L_{1}^{(\mathrm{s})}$ for $\left(h_{1}, h_{2}, h_{3}\right)=(1 \mathrm{e}-3,1 \mathrm{e}-2,1 \mathrm{e}-1)$.

Unlike in the high-frequency sampling framework, however, Algorithm 1 may be inefficient when $\Delta$ is large, with the worst acceptance rate being $e^{a b^{\alpha} \Gamma(-\alpha)}$. It is worth comparing Algorithm 1 with the double rejection sampling algorithm recently developed in Devroye [10], in expected time not depending upon all the model parameters.

\section{Algorithm 2.}

Step 1 Set

$$
\begin{aligned}
& \xi_{1} \leftarrow a\left(1-e^{-\alpha \lambda \Delta}\right) b^{\alpha} \Gamma(2-\alpha), \\
& \xi_{2} \leftarrow 1+\frac{2+\sqrt{\pi / 2}}{\pi} \sqrt{2 \xi_{1}}, \\
& \xi_{3} \leftarrow \sqrt{\frac{\pi}{2}} e^{-\frac{\xi_{1} \pi^{2}}{8}}\left(\xi_{2}-1\right) .
\end{aligned}
$$

Step 2 Generate $U_{1}$ and $U_{2}$ as independent uniform $(0,1)$. If $\xi_{1}<1$, then go to Step 4. 
Step 3 If $U_{1}<\frac{\xi_{2}}{\xi_{2}+2 \sqrt{2 \xi_{1}} \xi_{3}}$, then generate $Z_{1}$ as $\mathcal{N}(0,1)$ and set $V \leftarrow\left|Z_{1}\right| / \sqrt{\xi_{1}}$. Otherwise, set $V \leftarrow \pi\left(1-U_{2}^{2}\right)$. Go to Step 5 .

Step 4 If $U_{1}<\frac{\sqrt{\pi} \xi_{2}}{\sqrt{\pi} \xi_{2}+2 \xi_{3}}$, then set $V \leftarrow \pi U_{2}$. Otherwise, set $V \leftarrow \pi\left(1-U_{2}^{2}\right)$.

Step 5 Set

$$
\begin{aligned}
& \xi_{4} \leftarrow \sqrt{\sin (V)\left(\frac{\alpha}{\sin (\alpha V)}\right)^{\alpha}\left(\frac{(1-\alpha)}{\sin ((1-\alpha) V)}\right)^{1-\alpha}}, \\
& \xi_{5} \leftarrow\left(\sqrt{\xi_{1}}+\alpha \xi_{4}\right)^{\frac{1}{\alpha}} \\
& \xi_{6} \leftarrow \frac{\xi_{5}}{\xi_{5}-\sqrt[2 \alpha]{\xi_{1}}}, \\
& \xi_{7} \leftarrow \frac{\pi e^{-\frac{\xi_{1}\left(1-\xi_{4}^{-2}\right)}{\alpha(1-\alpha)}}}{\left(1+\sqrt{\frac{\pi}{2}}\right) \sqrt{\xi_{1} / \xi_{4}}+\xi_{6}}\left[\xi_{2} e^{-\frac{\xi_{1} V^{2}}{2}} \mathbf{1}\left(V \geq 0, \xi_{1} \geq 1\right)\right. \\
&\left.\quad+\frac{\xi_{3}}{\sqrt{\pi-V}} \mathbf{1}(V \in(0, \pi))+\xi_{2} \mathbf{1}\left(V \in[0, \pi], \xi_{1}<1\right)\right] .
\end{aligned}
$$

Step 6 Generate $U_{3}$ as uniform $(0,1)$. If $V<\pi$ and $\xi_{7} U_{3} \leq 1$, then go to Step 7 . Otherwise, go to Step 2.

Step 7 Generate $U_{4}$ as uniform $(0,1)$ and set

$$
\begin{array}{lll}
\xi_{8} \leftarrow \xi_{4}^{\frac{2}{\alpha-1}} \alpha^{\frac{\alpha}{1-\alpha}}(1-\alpha), & \xi_{9} \leftarrow \frac{(1-\alpha)^{\alpha-1} \xi_{1}}{\alpha^{\alpha+1} \xi_{8}^{\alpha}}, \\
\xi_{10} \leftarrow \sqrt{\alpha \xi_{9} / \xi_{8}}, & \xi_{11} \leftarrow \xi_{10} \sqrt{\pi / 2}, \\
\xi_{12} \leftarrow \xi_{6} / \xi_{8}, & \xi_{13} \leftarrow \xi_{10}+\xi_{11}+\xi_{12} .
\end{array}
$$

Step 8 If $U_{4}<\xi_{11} / \xi_{13}$, then generate $Z_{2}$ as $\mathcal{N}(0,1)$, set $X \leftarrow \xi_{9}-\xi_{10}\left|Z_{2}\right|$, and go to Step 11.

Step 9 If $U_{4}<\left(\xi_{10}+\xi_{11}\right) / \xi_{13}$, then generate $U_{5}$ as uniform $\left[\xi_{9}, \xi_{9}+\xi_{10}\right]$, set $X \leftarrow U_{5}$, and go to Step 11.

Step 10 Generate $E_{1}$ as $\operatorname{Exp}(1)$ and set $X \leftarrow \xi_{9}+\xi_{10}+\xi_{12} E_{1}$. 
Step 11 Generate $E_{2}$ as $\operatorname{Exp}(1)$. If $X \geq 0$ and

$$
\begin{aligned}
\xi_{8}\left(X-\xi_{9}\right) & +\left(\frac{\xi_{1}}{\alpha(1-\alpha)}\right)^{\frac{1}{\alpha}}\left(X^{\frac{\alpha-1}{\alpha}}-\xi_{9}^{\frac{\alpha-1}{\alpha}}\right) \\
& -\frac{Z_{2}^{2}}{2} \mathbf{1}\left(X<\xi_{9}\right)-E_{1} \mathbf{1}\left(X>\xi_{9}+\xi_{10}\right) \leq E_{2},
\end{aligned}
$$

then exit with $X^{\frac{\alpha-1}{\alpha}}$. Otherwise, go to Step 2.

The algorithm possesses a surprising feature; if $\xi_{1} \geq 1$, then the expected number of iterations is uniformly bounded as

$$
\xi_{2} \sqrt{\frac{\pi}{2 \xi_{1}}}+2 \xi_{3} \sqrt{\pi} \leq \sqrt{\frac{1}{2 \pi}}+\frac{2}{\sqrt{\pi}}+\frac{1}{\sqrt{2}}+\frac{8}{\pi \sqrt{e}}+\sqrt{\frac{8}{\pi e}} \approx 4.7468288,
$$

while if $\xi_{1} \leq 1$, then

$$
2 \xi_{3} \sqrt{\pi}+\xi_{2} \pi \leq \frac{8}{\pi \sqrt{e}}+\sqrt{\frac{8}{\pi e}}+\sqrt{8}+\sqrt{\pi}+1 \approx 8.1132815,
$$

the upper bounds being valid regardless of the model parameters $(\alpha, a, b, \lambda, \Delta)$. In the case Algorithm 1 is less efficient, that is, when in terms of acceptance rates,

$$
\begin{array}{r}
\exp \left[a\left(1-e^{-\alpha \lambda \Delta}\right) \Gamma(-\alpha) b^{\alpha}\right] \\
=\exp \left[-\frac{\xi_{1}}{\alpha(1-\alpha)}\right]< \begin{cases}\left(\xi_{2} \sqrt{\frac{\pi}{2 \xi_{1}}}+2 \xi_{3} \sqrt{\pi}\right)^{-1}, & \text { if } \xi_{1} \geq 1, \\
\left(2 \xi_{3} \sqrt{\pi}+\xi_{2} \pi\right)^{-1}, & \text { if } \xi_{1} \leq 1,\end{cases}
\end{array}
$$

it is worth employing Algorithm 2. To be more illustrative, we provide Figure 1 to compare acceptance rates of Algorithm 1 and 2 against sampling frequency $\Delta$, where the model parameters are fixed $(a, b, \lambda)=(1.0,1.0,0.5)$. (This parameter setting will be used for numerical illustrations shortly in Section 5.) Acceptance rates of Algorithm 1 increases to 1 as $\Delta \downarrow 0$ and decreases to $e^{a b^{\alpha} \Gamma(-\alpha)}$ as $\Delta \uparrow+\infty$. In those parameter settings, Algorithm 2 outperforms when stepsize $\Delta$ is roughly greater than $4.0,2.5,1.0$, respectively, for $\alpha=0.4,0.6,0.8$. In any event, a decision can be made between Algorithm 1 and 2 simply based on (4.2) prior to implementation.

Straightforward decompositions of the random variable $\eta_{0}(\Delta)$ may do the job as well. Observe that its Lévy measure can be decomposed, for example, as

$$
w_{\Delta, 1}(z)=\sum_{k=1}^{n} \frac{a}{n}\left(1-e^{-\alpha \lambda \Delta}\right) z^{-1-\alpha} e^{-b z}, \quad n \in \mathbb{N},
$$



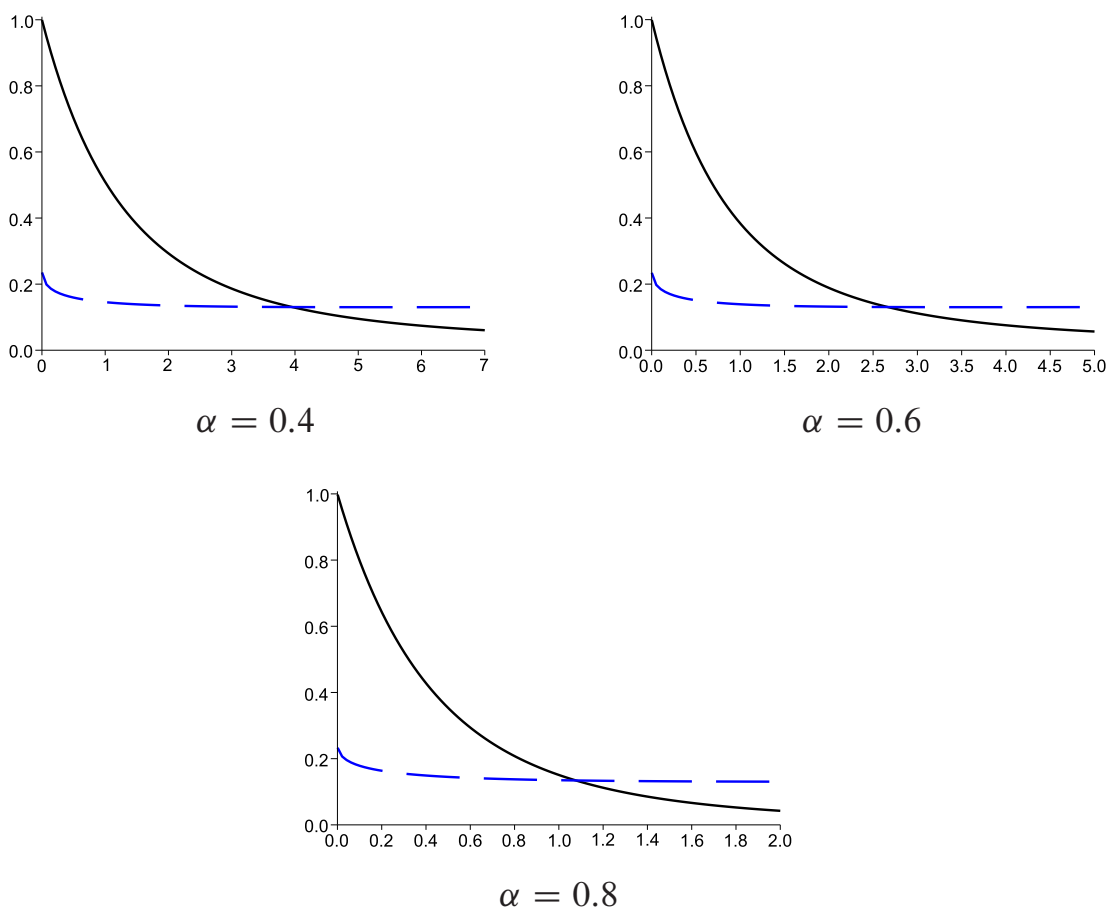

Figure 1. Acceptance rate ( $y$-axis) against sampling frequency $\Delta$ ( $x$-axis) of Algorithm 1 (solid lines) and Algorithm 2 (dotted lines). The model parameters are set $(a, b, \lambda)=(1.0,1.0,0.5)$.

that is, decomposing $\eta_{0}(\Delta)$ into $n$ iid random variables, due to its infinite divisibility. The decomposition (4.3) induces an algorithm consisting of $n$ independent runs of Algorithm 1 with a different parameter set, which results in the expected number $n e^{-a\left(1-e^{-\alpha \lambda \Delta}\right) \Gamma(-\alpha) b^{\alpha} / n}\left(=: n e^{n_{*} / n}=: H(n)\right)$ of loops in total, relative to the original expected number $e^{n_{*}}$ of Algorithm 1. Considering that $n$ is an integer, we can show that if both $n_{*}>1$ and $H\left(\left\lfloor n_{*}\right\rfloor\right) \wedge H\left(\left\lceil n_{*}\right\rceil\right)<e^{n_{*}}$ hold true, then the decomposition (4.3) provides a more efficient algorithm with $n=\left\lceil n_{*}\right\rceil$ if $H\left(\left\lceil n_{*}\right\rceil\right)<H\left(\left\lfloor n_{*}\right\rfloor\right)$ or with $n=\left\lfloor n_{*}\right\rfloor$ otherwise. The criterion can be checked prior to implementation and tends to hold in the low-frequency sampling framework. It is noteworthy that various different decompositions are certainly available, such as

$$
w_{\Delta, 1}(z)=\sum_{k=1}^{n} a e^{-\alpha \lambda(k-1) / n}\left(1-e^{-\alpha \lambda \Delta / n}\right) z^{-1-\alpha} e^{-b z},
$$

while the above simplest one (4.3) seems most effective for our purpose. 
Remark 4.2. Let $X_{S}$ and $X_{\mathrm{TS}}$ be random variables respectively with distributions $S(\alpha, a)$ under the probability measure $\mathbb{Q}$ and $\operatorname{TS}(\alpha, a, b)$ under $\mathbb{P}$. It is a straightforward application of Theorem 33.3 of Sato [21] to evaluate an expected value related to tempered stable random variables by the density transform

$$
\mathbb{E}_{\mathbb{P}}\left[\Phi\left(X_{\mathrm{TS}}\right)\right]=\mathbb{E}_{\mathbb{Q}}\left[\left.\frac{d \mathbb{P}}{d \mathbb{Q}}\right|_{\mathscr{g}} \Phi\left(X_{S}\right)\right],
$$

with $\Phi: \mathbb{R}_{+} \rightarrow \mathbb{R}$ such that $\mathbb{E}_{\mathbb{P}}\left[\left|\Phi\left(X_{\mathrm{TS}}\right)\right|\right]<+\infty$. Here, the Radon-Nykodym derivative is given in closed form $\left.(d \mathbb{P} / d \mathbb{Q})\right|_{\mathscr{g}}=e^{-b X_{S}} / \mathbb{E}_{\mathbb{Q}}\left[e^{-b X_{S}}\right]$, $\mathbb{Q}$-a.s., where $\mathcal{E}$ is the minimal $\sigma$-field generated by the random variable $X_{S}$. (This density transform formulation is found useful in the computation of Greeks under an asset price model driven by tempered stable processes. See Kawai-Takeuchi [17] for details.) This method does not employ acceptance-rejection sampling. (In fact, the tempered stable random variable $X_{\mathrm{TS}}$ is not even generated in this framework.) However, this is only valid for the evaluation of expectations, and the estimator variance $\operatorname{Var}_{\mathbb{Q}}\left(\left.(d \mathbb{P} / d \mathbb{Q})\right|_{\mathscr{E}} \Phi\left(X_{S}\right)\right)$ is typically greater than the original one $\operatorname{Var}_{\mathbb{P}}\left(\Phi\left(X_{\mathrm{TS}}\right)\right)$, provided that both variances are well defined, due to

$$
\mathbb{E}_{\mathbb{Q}}\left[e^{-b X_{\mathrm{TS}}}\right] \ll \mathbb{E}_{\mathbb{P}}\left[e^{-b X_{S}}\right] .
$$

Those observations discourage the use of this approach in the Monte Carlo framework.

We next consider simulation of the compound Poisson component. (We do not consider generation of the Poisson random variable $N(\Delta)$ here since its random number generator is available in most mathematical tools. See, for example, [1] for efficient Poisson generators.) Recall that $\left\{\eta_{k}(\Delta)\right\}_{k \in \mathbb{N}}$ has a common probability density $v_{\Delta}(x)$ given by (3.1). In a similar manner to Lemma 1 of [23], observe that

$$
v_{\Delta}(x) \leq \alpha \frac{e^{\lambda \Delta}-1}{e^{\alpha \lambda \Delta}-1}\left(\frac{b^{1-\alpha}}{\Gamma(1-\alpha)} x^{(1-\alpha)-1} e^{-b x}\right)=: C(\Delta) g_{1}(x), \quad x \in \mathbb{R}_{+},
$$

where $C(\Delta):=\alpha\left(e^{\lambda \Delta}-1\right) /\left(e^{\alpha \lambda \Delta}-1\right) \geq 1$ and $g(x)$ is the density of the gamma distribution $\Gamma(1-\alpha, b)$. Also, it holds that

$$
\frac{v_{\Delta}(x)}{C(\Delta) g_{1}(x)}=\frac{1-e^{b\left(1-e^{\lambda \Delta}\right) x}}{b x}=: g_{2, \Delta}(x), \quad x \in \mathbb{R}_{+} .
$$

This suggests the following acceptance-rejection sampling algorithm for the simulation of the random variable $\eta_{1}(\Delta)$. 


\section{Algorithm 3.}

Step 1 Generate $U$ as uniform $(0,1)$ and $V$ as $\Gamma(1-\alpha, b)$.

Step 2 If $U \leq g_{2, \Delta}(V)$, let $\eta_{1}(\Delta) \leftarrow V$. Otherwise, return to Step 1.

Acceptance rate here is $1 / C(\Delta)$ and is close to 1 when $\Delta$ is very small. In other words, Algorithm 3 is efficient in the high-frequency sampling framework. The acceptance rate may be small, however, when the stability index $\alpha$ is extremely close to zero along with a large stepsize $\Delta$. To address this issue, we can apply a simple yet very efficient improvement, due to [23], based on the composition method (see, for example, Devroye [9]) and the decomposition of the density function $v_{\Delta}(x)$ into an arbitrary number of density functions within the same class; for each $n \in \mathbb{N}$,

$$
v_{\Delta}(x)=\sum_{k=0}^{n-1} p_{n}(k)\left(e^{\lambda \Delta k / n} v_{\Delta / n}\left(e^{\lambda \Delta k / n} x\right)\right),
$$

where

$$
p_{n}(k):=\frac{\left(e^{\alpha \lambda \Delta / n}-1\right) e^{\alpha \lambda \Delta k / n}}{e^{\alpha \lambda \Delta}-1}, \quad k=0, \ldots, n-1 .
$$

Note that $p_{n}(k)>0$ and $\sum_{k=0}^{n-1} p_{n}(k)=1$ and that $e^{\lambda \Delta k / n} v_{\Delta / n}\left(e^{\lambda \Delta k / n} x\right)$ in the above summand acts as probability density function of $e^{-\lambda \Delta k / n} \eta_{1}(\Delta / n)$, which can be generated exactly by Algorithm 3 . The improved algorithm is as follows.

\section{Algorithm 4.}

Step 1 Generate $U$ as uniform $(0,1)$ and find the index

$$
j=\min \left\{l: \sum_{k=0}^{l} p_{n}(k) \geq U\right\} .
$$

Step 2 Generate a random variable $\eta_{1}(\Delta / n)$ by Algorithm 3 .

Step 3 Return $e^{-\lambda \Delta j / n} \eta_{1}(\Delta / n)$.

Relative to Algorithm 3, acceptance rate increases to $1 / C(\Delta / n)$ from $1 / C(\Delta)$. It can be made as close to 1 as one wishes by increasing $n$ at almost no cost; additional computing effort is required at Step 1, while this is a minor increase.

Remark 4.3. With those exact simulation methods for the tempered stable distribution, we can readily derive an exact simulation algorithm for the multivariate, 
possibly skewed, normal tempered stable Lévy process [4]. To be precise, the normal tempered stable Lévy process $\left\{Y_{t}: t \geq 0\right\}$ in $\mathbb{R}^{d}$ is defined by

$$
Y_{t}=\mu t+\beta \Lambda L_{t}^{(\mathrm{ts})}+\Lambda^{1 / 2} B_{L_{t}^{(\mathrm{ts})}}
$$

where $\mu, \beta \in \mathbb{R}^{d}$, the matrix $\Lambda \in \mathbb{R}^{1 \times d}$ is symmetric positive definite and has unit determinant, and $\left\{B_{t}: t \geq 0\right\}$ is a standard Brownian motion in $\mathbb{R}^{d}$ (see [2,4] for details.) That is to say, the law $\mathscr{L}\left(Y_{t}\right)$ can be simulated immediately from the law $\mathscr{L}\left(L_{t}^{(\mathrm{ts})}\right)$ and the standard normal distribution in $\mathbb{R}^{d}$. To the best of our knowledge, simulation of normal tempered stable Lévy processes has been discussed solely through infinite series representations, such as (2.8).

We close this section with discussing simulation of random elements arising in Corollary 3.2 above. In principle, the recipe is almost the same, that is, Algorithm 1 is applicable to $\eta_{0}^{+}(\Delta)$ and $\eta_{0}^{-}(\Delta)$, while Algorithm 3 to $\left\{\eta_{k}^{+}(\Delta)\right\}_{k \in \mathbb{N}}$ and $\left\{\eta_{k}^{-}(\Delta)\right\}_{k \in \mathbb{N}}$. When $\alpha_{+}=\alpha_{-}=: \alpha$, however, we can go a little further. The proposal distribution for $\eta_{0}^{+}(\Delta)+\eta_{0}^{-}(\Delta)$ is then again a stable distribution, which can be simulated as a single stable random variable. Write

$$
\eta(\Delta):=\eta_{0}^{+}(\Delta)+\eta_{0}^{-}(\Delta),
$$

where $\eta_{0}^{ \pm}(\Delta) \sim \operatorname{TS}\left(\alpha, \widetilde{a}_{ \pm}, b_{ \pm}\right), \widetilde{a}_{ \pm}:=a_{ \pm}\left(1-e^{-\alpha \lambda \Delta}\right)$, and moreover

$$
c:=e^{-\Gamma(-\alpha)\left(1-e^{-\alpha \lambda \Delta}\right)\left(a_{+} b_{+}^{\alpha}+a_{-} b_{-}^{\alpha}\right)} .
$$

The density $f_{\eta(\Delta)}(x)$ of the random variable $\eta(\Delta)$ has a upper bound as

$$
\begin{aligned}
f_{\eta(\Delta)}(x) & =\int_{\mathbb{R}} f_{\mathrm{TS}\left(\alpha, \widetilde{a}_{+}, b_{+}\right)}(x-y) f_{\mathrm{TS}\left(\alpha, \widetilde{a}_{-}, b_{-}\right)}(-y) d y \\
= & c \int_{-\infty}^{x \wedge 0} e^{-b_{+}(x-y)} e^{b_{-} y} f_{S\left(\alpha, \widetilde{a}_{+}\right)}(x-y) f_{S\left(\alpha, \widetilde{a}_{-}\right)}(-y) d y \\
\leq & c e^{-b_{+} x} e^{\left(b_{+}+b_{-}\right)(x \wedge 0)} \int_{-\infty}^{x \wedge 0} f_{S\left(\alpha, \widetilde{a}_{+}\right)}(x-y) f_{S\left(\alpha, \widetilde{a}_{-}\right)}(-y) d y \\
= & c\left(e^{-b_{+} x} \mathbf{1}(x>0)+e^{-b-|x|} \mathbf{1}(x<0)\right) \\
\quad & \quad \times \int_{\mathbb{R}} f_{S\left(\alpha, \widetilde{a}_{+}\right)}(x-y) f_{S\left(\alpha, \widetilde{a}_{-}\right)}(-y) d y \\
= & : c g_{3}(x) \int_{\mathbb{R}} f_{S\left(\alpha, \widetilde{a}_{+}\right)}(x-y) f_{S\left(\alpha, \widetilde{a}_{-}\right)}(-y) d y,
\end{aligned}
$$


where the last integral is the density of the (bilateral) stable distribution with no drift, $S\left(\alpha, \widetilde{a}_{+}, \widetilde{a}_{-}\right)$say, with Lévy density

$$
\frac{\widetilde{a}_{+}}{z^{1+\alpha}} \mathbf{1}(z>0)+\frac{\widetilde{a}_{-}}{|z|^{1+\alpha}} \mathbf{1}(z<0) .
$$

This stable proposal distribution can be simulated in a similar manner to (2.3):

$$
\begin{aligned}
S\left(\alpha, \widetilde{a}_{+}, \widetilde{a}_{-}\right) \stackrel{\mathscr{L}}{=}\left(\frac{-\left(\widetilde{a}_{+}+\widetilde{a}_{-}\right) \Gamma(-\alpha) \cos (\pi \alpha / 2)}{\cos (V)\left(1+(\beta \tan (\pi \alpha / 2))^{2}\right)^{-1 / 2}}\right)^{\frac{1}{\alpha}} & \\
& \times \sin (\alpha(V+\theta))\left(\frac{\cos (V-\alpha(V+\theta))}{E}\right)^{\frac{1-\alpha}{\alpha}},
\end{aligned}
$$

where $V$ is a uniform random variable on the interval $(-\pi / 2, \pi / 2), E$ is a standard exponential random variable independent of $V, \beta:=\left(\widetilde{a}_{+}-\widetilde{a}_{-}\right) /\left(\widetilde{a}_{+}+\widetilde{a}_{-}\right)$and $\theta:=\arctan (\beta \tan (\pi \alpha / 2)) / \alpha$. (See Chambers et al. [8] for details.) In that case, we can apply the following acceptance-rejection sampling algorithm, similar to Algorithm 1 above, to generate $\eta(\Delta)$ with the bilateral stable proposal distribution $S\left(\alpha, \widetilde{a}_{+}, \widetilde{a}_{-}\right)$.

\section{Algorithm 5.}

Step 1 Generate $U$ as uniform $(0,1)$ and $V$ as $S\left(\alpha, \widetilde{a}_{+}, \widetilde{a}_{-}\right)$.

Step 2 If $U \leq g_{3}(V)$, let $\eta(\Delta) \leftarrow V$. Otherwise, return to Step 1.

The acceptance rate at Step 2 is $c^{-1}=e^{\Gamma(-\alpha)\left(1-e^{-\alpha \lambda \Delta}\right)\left(a_{+} b_{+}^{\alpha}+a_{-} b_{-}^{\alpha}\right)}$, which increases to 1 as $\Delta$ tends to zero. An important remark here is that generation of $\eta(\Delta)$ by Algorithm 5 above may not always outperform that of $\eta_{+}(\Delta)$ and $\eta_{-}(\Delta)$ through implementation of Algorithm 1 twice. To describe this, fix

$$
\alpha_{ \pm}=\alpha \in(0,1) \text { and } a_{ \pm}=b_{ \pm}=1,
$$

for simplicity. Also, let $N_{1}(\lambda \Delta)$ and $N_{5}(\lambda \Delta)$ be the expected loop numbers required, respectively, for generation of $\eta_{+}(\Delta)$ and $\eta_{-}(\Delta)$ by Algorithm 1 twice and for generation of $\eta(\Delta)$ by Algorithm 5 once, where $N_{1}(s):=2 e^{-\left(1-e^{-\alpha s}\right) \Gamma(-\alpha)}$ and $N_{5}(s):=e^{-2\left(1-e^{-\alpha s}\right) \Gamma(-\alpha)}$. Then, observe that

$$
N_{1}(s)-N_{5}(s) \begin{cases}\geq 0, & \text { if } s \in\left[0,-\frac{1}{\alpha} \ln \left(1+\frac{\ln 2}{\Gamma(-\alpha)}\right)\right], \\ <0, & \text { otherwise. }\end{cases}
$$

Hence, Algorithm 5 is of practical use when $\Delta \leq-(\lambda \alpha)^{-1} \ln (1+\ln 2 / \Gamma(-\alpha))$ and is preferable for small $\alpha$ since the boundary point is strictly decreasing in $\alpha$ towards zero. 


\section{Numerical illustration}

We provide in Figure 2 typical sample paths of finite variation tempered stable Ornstein-Uhlenbeck processes in the high-frequency sampling framework, based on the exact transitions given in Theorem 3.1 and the acceptance-rejection sampling methods described in Algorithms 1 and 3 above. The model parameters are set $(a, b, \lambda)=(1.0,1.0,0.5)$ and $\alpha=0.4,0.6$ and 0.8 , the same setting for Figure 1 . For simplicity, we set the initial state

$$
Y_{0}=a \Gamma(1-\alpha) b^{\alpha-1}=\lim _{t \uparrow+\infty} \mathbb{E}\left[Y_{t}\right],
$$

that is the mean of the stationary distribution $\operatorname{TS}(\alpha, a, b)$. Sample paths are generated over time intervals $[0,100]$ and $[0,200]$, where stepsize is $\Delta=0.1$. Hence, 1000 and 2000 recursive increments are needed, respectively, for the intervals $[0,100]$ and $[0,200]$. In the context of asymptotic statistics for discretely observed Ornstein-Uhlenbeck processes, it is often preferable to take $\Delta$ small and $T$ large, as in this setting. (See, for example, $[7,13]$.)

Computing times required for an implementation of 2000 recursive increments by $\mathrm{R}$ software are $0.20,0.25$ and 0.34 seconds, respectively, for $\alpha=0.4,0.6$ and 0.8 , on a typical desktop PC. (Computing times can be reduced much further by using a low-level language such as $\mathrm{C}$, rather than high-level ones such as $\mathrm{R}$ and MATLAB.) In principle, the difference in computing time comes from acceptance rates in Algorithms 1 and 3. In our parameter setting, acceptance rates in Algorithm 1 (one sample from $\mathscr{L}\left(\eta_{0}(\Delta)\right)$ ) are $0.929,0.896$ and 0.800 , respectively, while in Algorithm 3 (one sample from $\mathscr{L}\left(\eta_{1}(\Delta)\right)$ ), the acceptance rates are, respectively, $0.985,0.990$ and 0.995 . Clearly, acceptance rate for $\eta_{0}(\Delta)$ virtually dominates that for $\eta_{1}(\Delta)$, due to very small means $0.074,0.109$ and 0.225 of the Poisson random variable $N(\Delta)$.

Finally, let us comment in brief on the existing simulation method based on the infinite shot noise series representation (2.12) for comparison. The simulation use of infinite series representations entails a finite truncation of infinite sum. (See Imai and Kawai [11].) We have observed through numerical experiments that under the same parameter setting as above, approximately 4000 summands are needed to obtain sensible sample paths over time interval $[0,200]$. (Our observation here is based upon Monte Carlo estimation of the mean $\mathbb{E}\left[Y_{200}\right] \approx Y_{0}$ and the variance $\operatorname{Var}\left(Y_{200}\right) \approx a \Gamma(2-\alpha) b^{\alpha-2}$.) Although results are different for different parameter settings and for different criteria and although generalizing solely based on numerical experiments is somewhat risky, it seems fair to claim that our exact simulation algorithm outperforms, considering many kinds of random sequences to be generated and all the other operations such as taking minimum, sorting the series 

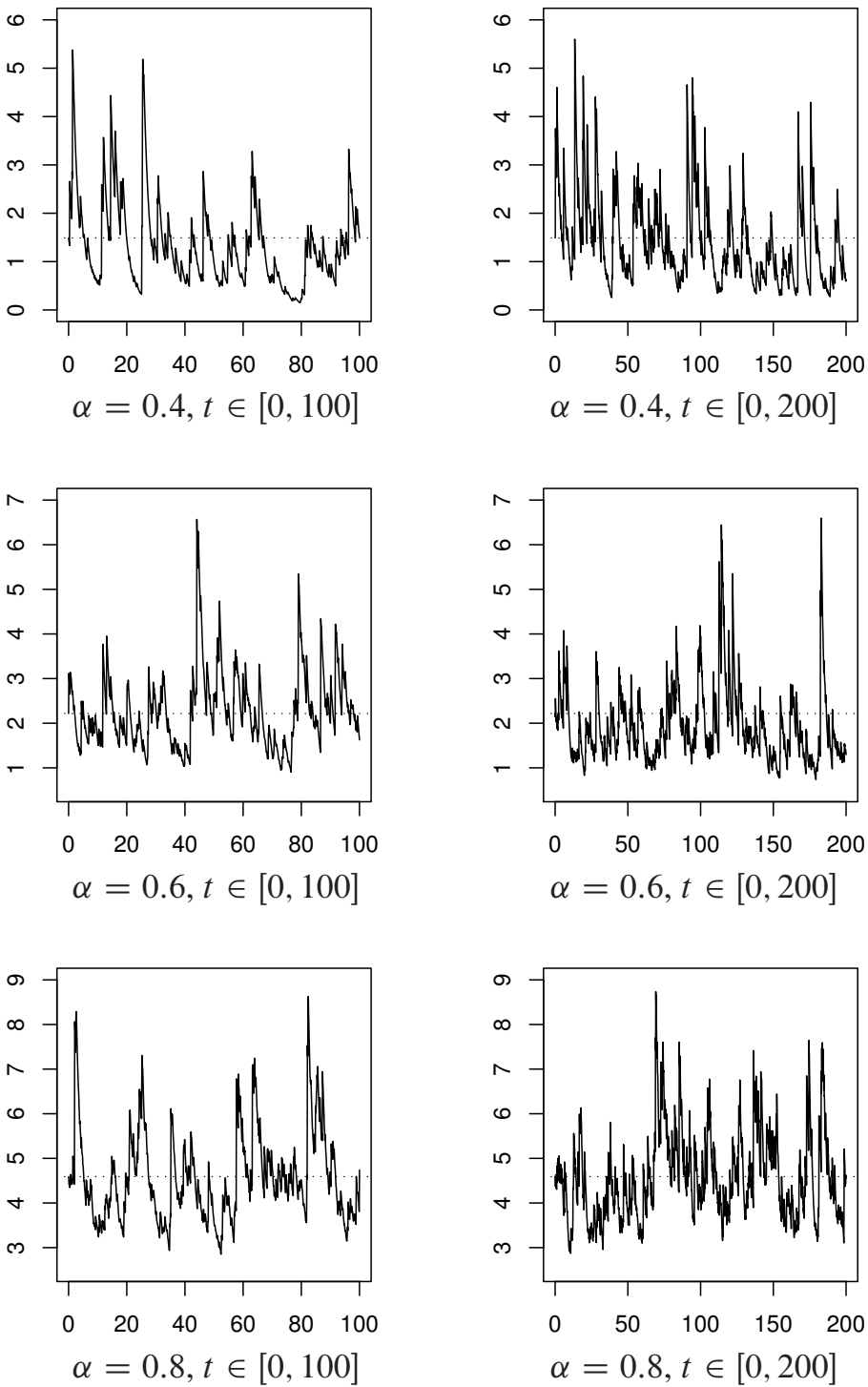

Figure 2. The figures show typical sample paths of tempered stable Ornstein-Uhlenbeck processes through exact simulation algorithm. The model parameters are set $Y_{0}=a \Gamma(1-\alpha) b^{\alpha-1}\left(=\lim _{t \uparrow+\infty} \mathbb{E}\left[Y_{t}\right]\right),(a, b, \lambda)=(1.0,1.0,0.5)$. The horizontal dashed lines indicate the initial state $Y_{0}$. 
by $\left\{T_{k}\right\}_{k \in \mathbb{N}}$, counting arrivals $\left\{\widetilde{\Gamma}_{k}\right\}_{k \in \mathbb{N}}$ and monitoring at every discrete time point in the representation (2.12). In particular, some of those operations may require a tremendous amount of computing time in high-level languages based on matrix operations.

\section{Concluding remarks}

In this paper, we have discussed exact simulation algorithms for a wide class of Ornstein-Uhlenbeck processes of finite variation tempered stable stationary laws based on the exact transition probability between consecutive observations. We have adopted acceptance-rejection sampling to simulate tempered stable and compound Poisson distributions, respectively, with stable and gamma proposal distribution. We have shown that Algorithms 1, 3 and 5 approach to perfect acceptancerejection sampling as stepsize tends to zero. This fact supports the proposed method for validation and estimation purposes under high-frequency sampling. We have also addressed the issue of their inefficient functionality when sampling frequency is very low. Our algorithms prove applicable to simulations of bilateral finite variation tempered stable Ornstein-Uhlenbeck processes and normal tempered stable processes as well. Our exact simulation algorithms work more efficiently relative to the existing approximative simulation method based on infinite series representation of sample paths. It is of practical interest to extend to the infinite variation setting, in which no practical exact simulation method is known yet. Those topics are addressed in subsequent papers $[15,16]$. It would also be an interesting future research topic to improve Algorithm 5 to a uniformly fast algorithm, in a similar spirit to Algorithm 3.

\section{Bibliography}

[1] J. H. Ahrens and U. Dieter, Computer generation of Poisson deviates from modified normal distributions, ACM Trans. Math. Software 8 (1982) no. 2, 163-179.

[2] O. E. Barndorff-Nielsen, J. Kent and M. Sørensen, Normal variance-mean mixtures and $z$-distributions, Int. Stat. Rev. 50 (1982), 145-159.

[3] O. E. Barndorff-Nielsen and N. Shephard, Non-Gaussian Ornstein-Uhlenbeck-based models and some of their uses in financial economics (with discussion), J.R. Stat. Soc. Ser. B Stat. Methodol. 63 (2001), no. 2, 167-241.

[4] O. E. Barndorff-Nielsen and N. Shephard, Normal modified stable processes, Theory Probab. Math. Statist. 65 (2002), 1-19.

[5] F. E. Benth, M. Gorth and R. Kufakunesu, Valuing volatility and variance swaps for a non-Gaussian Ornstein-Uhlenbeck stochastic volatility model, Appl. Math. Finance 14 (2007), no. 4, 347-363. 
[6] A. Brix, Generalized Gamma measures and shot-noise Cox processes, $\underline{A d v}$. in Appl. Probab. 31 (1999), no. 4, 929-953.

[7] P. J. Brockwell, R. A. Davis and Y. Yang, Estimation for nonnegative Lévy-driven Ornstein-Uhlenbeck processes, J. Appl. Probab. 44 (2007), no. 4, 977-989.

[8] J. M. Chambers, C. L. Mallows and B.W. Stuck, A method for simulating stable random variables, J. Amer. Statist. Assoc. 71(354) (1976), 340-344.

[9] L. Devroye, Non-Uniform Random Variate Generation, Springer-Verlag, New York, 1986.

[10] L. Devroye, Random variate generation for exponential and polynomially tilted stable distributions, ACM Trans. Model. Comput. Simul. 19 (2009), no. 4, Article No. 18.

[11] J. Imai and R. Kawai, On finite truncation of infinite shot noise series representation of tempered stable laws, to appear in Phys. A, DOI:10.1016/j.physa.2011.07.028.

[12] J. Imai and R. Kawai, Numerical inverse Lévy measure method for infinite shot noise series representation, preprint.

[13] G. Jongbloed, F. H. van der Meulen and A. W. van der Vaart, Nonparametric inference for Lévy-driven Ornstein-Uhlenbeck processes, Bernoulli 11 (2005), no. 5, 759-791.

[14] M. Kanter, Stable densities under change of scale and total variation inequalities, Ann. Probab. 3 (1975), no. 4, 697-707.

[15] R. Kawai and H. Masuda, Infinite variation tempered stable Ornstein-Uhlenbeck processes with discrete observations, to appear in Comm. Statist. Simulation Comput., DOI:10.1080/03610918.2011.582561.

[16] R. Kawai and H. Masuda, On simulation of tempered stable random variates, $\underline{\text { J. Com- }}$ put. Appl. Math. 235 (2011), no. 8, 2873-2887.

[17] R. Kawai and A. Takeuchi, Computation of Greeks for asset price dynamics driven by stable and tempered stable processes, to appear in Quant. Finance, DOI:10.1080/ 14697688.2011.589403.

[18] H. Masuda, On multidimensional Ornstein-Uhlenbeck processes driven by a general Lévy process, Bernoulli 10 (2004), no. 1, 1-24.

[19] J. R. Michael, W. R. Schucany and R. W. Haas, Generating random variates using transformations with multiple roots, Amer. Statist. 30 (1976), 88-90.

[20] J. Rosiński, Tempering stable processes, Stochastic Process. Appl. 117 (2007), no. 6, $677-707$.

[21] K. Sato, Lévy Processes and Infinitely Divisible Distributions, Cambridge University Press, Cambridge, 1999. 
[22] M. C. K. Tweedie, An index which distinguishes between some important exponential families, in: Statistics: Applications and New Directions, Proceedings of the Indian Statistical Institute Golden Jubilee International Conference, pp. 579-604, edited by J. Ghosh and J. Roy, Indian Statistical Institute, Calcutta, 1984.

[23] S. Zhang and X. Zhang, Exact simulation of IG-OU processes, Methodol. Comput. Appl. Probab. 10 (2008), no. 3, 337-355.

[24] S. Zhang and X. Zhang, On the transition law of tempered stable Ornstein-Uhlenbeck processes, J. Appl. Probab. 46 (2009), no. 3, 721-731.

[25] V. M. Zolotarev, One-Dimensional Stable Distributions, American Mathematical Society, Providence, RI, 1986.

Received January 26, 2011; revised August 10, 2011.

\section{Author information}

Reiichiro Kawai, Department of Mathematics, University of Leicester,

Leicester LE1 7RH, UK.

E-mail: reiichiro.kawai@le.ac.uk

Hiroki Masuda, Institute of Mathematics for Industry, Kyushu University, Fukuoka 819-0395, Japan.

E-mail: hiroki@imi.kyushu-u.ac.jp 